\title{
Kinetic Gas Molecule Optimization for MRI Brain Segmentation using the Fuzzy C-Means Clustering
}

\author{
V.Vinay Kumar, S. Kusumavathi, K.S. Sharma
}

\begin{abstract}
As of past due, bunching manner has have become out to be notable for particular scientists due to particular software fields like correspondence, a ways flung systems control, and biomedical region, and plenty of others. on this way, a extensive kind of research has simply been made with the aid of the scientists to accumulate an progressed calculation for grouping. one of the first-rate technique among the experts is an improvement that has been efficaciously used for grouping. on this paper, Kinetic fuel Molecule Optimization (KGMO) in view of centroid instatement for picture department carried out for the fluffy c-implies bunching (FCM). The proposed framework is moreover named as KGMO-KFCM-BIM. For MRI cerebrum tissue branch, KFCM is maximum pleasant system in view of its precision. The extensive constraint of the standard KFCM is peculiar centroids instatement, because of the truth which devours the execution time to reach at the best arrangement. an awesome manner to quicken the division technique, KGMO is carried out to instate the centroids of required companies. The quantitative proportions of consequences have been checked out utilizing the measurements, as an example, cube coefficient, Jaccard co-proficient and precision. the quantity of emphasess and managing of KGMO-KFCM-BIM approach take least esteem at the same time as contrasted with not unusual KFCM. The KGMO-KFCM-BIM method is fantastically efficient and quicker than regular KFCM for mind tissue department.
\end{abstract}

Watchwords-bunching, centroid introduction, Kernel fluffy C-implies (KFCM), Kinetic gasoline Molecule Optimization (KGMO), MRI mind tissue division.

\section{INTRODUCTION}

Cerebrum Tumors in the vital concerned device (CNS) are found in round 250000 sufferers each year over the arena [1]. the overall event of thoughts tumors has been represented round three.nine/one lac/year in male and three.0/one lac/yr in girls [2]. The South primary Asia district including India has a pace of one.5 and zero.7/one lac/one year in men and girls independently [3]. sufferers with mind disorder have confined survival time with inescapable repeat and consequent passing, and restorative technique is the critical line treatment. The middle survival time for sufferers with excessive-grade mind malignancy is ready 14 months, except singular survival is heterogeneous [4-5]. Be that as it may, medical method disbursed neurological deficiencies are related with a lot much less fortunate survival; as such, it's

Revised Version Manuscript Received on August 19, 2019.

V.Vinay Kumar, Electronics and communication, Anurag group of Institutions Hyderabad, Telangana, India

S. Kusumavathi, Electronics and communication, Anurag group of Institutions, Hyderabad, Telangana, India

K.S. Sharma, Electronics Engineering, Amrutvahini college of Engineering Sangamner, Maharashtra, India miles simple to perform broad resection of malignant growth tissue with out bargaining non-disease tissue [6-7]. numerous creative advances have made critical obligations in restorative approach intraoperative Magnetic Resonance Imaging (MRI) and Computed Tomography (CT), ultrasound, Raman spectroscopy, and fluorescence-guided resections; be that as it is able to, these advances have elements of interest and disadvanages in cerebrum disease acknowledgment [6-11].

To understand the thoughts malignant increase, department is the maximum troublesome difficulty in mild of the regular and anomalous systems in the cerebrum Tissues [12]. in this KGMO-KFCM-BIM framework, a grouping technique for department is taken into consideration. Bunching is a system of apportioning or amassing plenty of unlabeled articles into various corporations just so comparable devices are general to one business enterprise. The principle tactics in grouping are Crisp bunching (CC) (or tough Clustering (HC)) [13], FCM calculation, ghostly grouping [14], various leveled techniques (e.g., tree searching for approach) centered analyzing calculations (e.g., oneself finding out guide and Iterative Self-Organizing data analysis (ISODA) [15]), and conveyance based totally techniques (e.g., Expectation Maximization (EM) framework [16]), and thickness primarily based techniques [17], [18]. specifically, Density-primarily based spatial Clustering software program with commotion (DBSCRN) [9] and Ordering elements are applied to apprehend the bunching structure [19]. A everyday for the CC technique is that the restrict among bunches is completely characterised. Be that as it is able to, in unique actual cases, the boundaries amongst businesses cannot be in reality characterized. some examples may also moreover have an area with more than one bunch. In the instance grouping FC method offers a advanced bunching effect [20], [22]. FCM is an iterative calculation [23]. FCM is amazing at settling the ambiguities and vulnerabilities within the photo [24]. Be that as it can, FCM can not manage the strain in homogeneity and gradually difficult to lessen the commotion. the use of thoughts tumor acknowledgment is furnished via utilizing altered FCM framework. In that, a much undertaking spotlight vector place is implemented for department this is trailed by the thing deceives. The KFCM framework is broadened which fuses the location phrases into its goal work [25]. The goal of

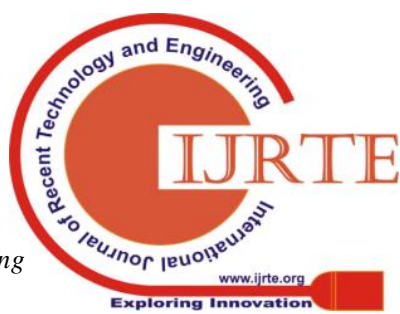


FCM is to discover institution centroids and that restriction the goal art work.

The KFCM is gotten from the primary FCM relying at the 'thing method' [26]. thru using factor deceives, the KFCM framework endeavors to address this problem by the use of mapping facts with nonlinear element extraction [27]. Enhancement calculations are supplied for grouping method. In development based bunching a large portion of the exploration is packed in the squared blunder and that they've applied some improvement techniques, as an example, Genetic calculation GA [28], PSO [29], bacterial searching enhancement [30] mimicked strengthening [31], counterfeit honey bee province [32] and Firefly gadget (FS) [33]. in this paper, KFCM centroid abnormal creation issue the KGMO-KFCM-BIM device is offered. The KGMO-KFCM-BIM method improves the department execution. The exhibition parameters are dice coefficient, Jaccard co-productive and exactness.

This paper is created as pursues. segment II reviews some ongoing papers on thoughts tumor reputation related techniques. In place III, KGMO-KFCM-BIM strategy. The section IV demonstrates the relative trial end result for cutting-edge and proposed device. The surrender is made in phase V.

\section{ASSOCIATED WORK}

Some inquires about are proposed by way of manner of analysts in mind tumor discovery. In this situation, the quick assessment of a few massive commitments to the modern-day writings are exhibited.

Ronghua Shang et al. [34] proposed a superior FCM framework clone piece spatial FCM (CKS-FCM). A resistant underlying bunch focuses, which empowers the intermingling global quality. The spatial information is covered the intention capability and CKS-FCM utilized a non-Euclidean separation depending on piece capacity to supplant the Euclidean separation. The number one confinement of the framework is Low department exactness and espresso heartiness.

Li Liu et al. [35] proposed a completely unique hearty dataset characterization technique dependent on Neighbor searching and FCM (NSKFCM) frameworks to decrease the impact of parameters vulnerabilities with dataset order. a few streamlined systems, inclusive of neighbor looking, controlling grouping shape and flexible separation part work, are applied to recognize the problems of diverse bunches, the stability, and consistency of order, separately. Numerical analyses at lengthy ultimate display the attainability and energy of the proposed method. The NSKFCM framework gadgets aside furthermore managing try and factor the photos.

Elazab et al. [36] have displayed department of mind tissues from MRI using versatile regularized detail primarily based FCM. That framework turn out to be grouped into three stages, as an instance, everyday channel, center channel, and formulated weighted image. The frameworks control the heterogeneity of grayscales inside the area and enterprise this measure for close by logical statistics and supplant the same vintage Euclidean separation with Gaussian outspread premise detail works. The essential blessings are adjusted to clone framework modified into carried out to enhance the

community setting, superior strength that jam photograph subtleties, autonomy of bunching parameters, and faded computational prices, however the principle shortcoming is decrease entropy.

Y.T. Chen et al. [37] have proposed impartial phase examination primarily based totally kernelized fluffy by way of way of utilizing MIS. they've cited the branch execution of six techniques (ok-implies, FCM, KFCM, ICFCM, KWFLICM, and ICKFCM) for the mimicked MRI photographs in silent case, commotion case, and real medicinal images. The number one disadvantage of the framework is plenty less precise.

Li, Haiyang et al. [38] added a framework for MIS, referred to as Dynamic PSO and ok-implies grouping framework (DPSOK). Dynamic PSO (DPSO) and ok-implies grouping approach become the bottom of the DPSOK framework. They made DPSOK a respectable improved calculation with the useful resource of upgrading the figuring approach of its dormancy weight and gaining knowledge of factorsExperimentation effects tested that DPSOK calculation can successfully improve the k-implies framework's global inquiry ability. DPSOK calculation conveyed better effects in enhancing photo department first-rate and skillability contrasted with traditional PSO okay-implies framework.

to overcome the formerly mentioned downsides, KGMO-KFCM-BIM calculation is actualized for upgrading the exhibition of thoughts tumor branch and identification.

\section{KGMO-KFCM-BIM TECHNIQUE \& RESULTS}

Picture department assumes a noteworthy activity in some continuous packages, for example, layout acknowledgment, photograph coding, laptop imaginative and prescient and medicinal photo studies. on this paper, KGMO-KFCM-BIM calculation is obtainable for MRI cerebrum tissue department, this is appeared inside the Fig. 1.

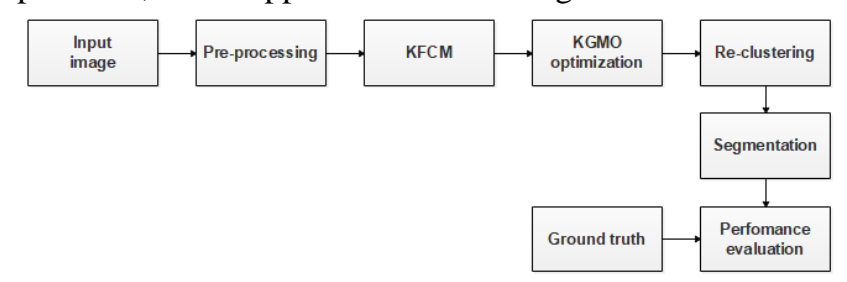

Fig. 1. KGMO-KFCM-BIM block diagram

The aspect of KGMO-KFCM-BIM technique is to discover the organization focuses that restriction a specialty (goal) capability of FCM. through using iteratively refreshing the bunch focuses and the enrollment grade for each datum factor, FCM iteratively actions the employer focuses "to as a minimum one side" region interior an informational series. Be that as it can, it is unrealistic to discover an outstanding affiliation in a outstanding time. The working of the FCM relies upon on the underlying centroids so the selection of the centroids is maximum extensive element inside the FCM. for this reason, on this paper the KGMO-KFCM-BIM method is a programmed centroid willpower relying on KGMO for FCM. The proposed approach contains modules, the KGMO 
bunching module and the FCM grouping module. in the underlying degree, the KGMO bunching module is completed for a quick length for programmed grouping, framing round or near spherical form information bunches. The final results from the KGMO grouping module is applied due to the fact the underlying seed of the FCM module. The superior fragments are Contour with the aid of Geometric dynamic shape shape (GACF). here, the wellbeing really worth decided and the fine health well really worth is consistent as a centroid esteem. through utilising the properly being esteem, the KFCM division is dealt with ultimately, the presentation is determined.

\section{A. KGMO enhancement}

It's far supplied with the aid of utilising Boyle, Charles and gay-Lussac gasoline legal guidelines, which depend on trial perceptions to painting the in reality seen nature of fuel atoms. The fuel atom development is based upon positive properties, as an instance, weight, temperature and quantity of the fuel. The idea of the particles in a gas become portrayed via five hypothesizes and dynamic atomic speculation of the first-rate gases as pursues:

- A fuel carries of a meeting of little atoms that flow in straight line movement. The development is based upon on Newton's laws.

- The atoms in a gas has no volume. They resemble a factor.

- The crashes among atoms are perfectly flexible. power can be neither picked up nor out of place during a crash.

- there's no attractive or appalling strength amongst particles

- The normal motor power of an atom is $3 \mathrm{kT} / 2$, wherein $\mathrm{T}$ is the best temperature, and good enough is the Boltzmann steady, which has the estimation of $1.38 \times 10-23 \mathrm{~m} 2 \mathrm{~kg} \mathrm{~s}-2$ adequate- 1 .

accept, that the framework consists of debris. The location of the professional is depicted via way of Eq. (1),

$$
Y_{j}=\left(y_{j}^{1}, \ldots \ldots ., y_{j}^{d}, \ldots \ldots . y_{j}^{m}\right) \quad \text { for }(j=1,2, \ldots . ., M)
$$

Where defines the location of the agent in the th dimension. The velocity of the agent expressed as below Eq. (2):

$$
V_{j}=\left(v_{j}^{1}, \ldots \ldots ., v_{j}^{d}, \ldots \ldots . v_{j}^{m}\right) \quad \text { for }(j=1,2, \ldots ., M)
$$

(2)

Where represents the agent velocity in the dimension.

The movement of the particles depends on the Boltzmann distribution that means the velocity of the random particle is proportional to the particle's kinetic energy. The kinetic energy of the particle is expressed as Eq. (3),

$$
\begin{array}{r}
k_{j}^{d}(u)=\frac{3}{2} M b T_{j}^{d}(u), K_{j}=\left(k_{j}{ }_{j}, \ldots \ldots ., k_{j}^{d}, \ldots \ldots . k_{j}^{m}\right) \\
\quad f o r(j=1,2, \ldots . ., M)
\end{array}
$$

Where a total number of particles is represented as is the Boltzmann constant and the temperature of the agent in the dimension at a time is represented as . The molecules velocity is updated by Eq. (4).

$$
\begin{aligned}
{v_{j}^{d}}_{j}(u+1)= & T_{d}^{j}(u) w v_{j}^{d}(u) \\
& +D_{1} \operatorname{rand}_{j}(u)\left(\operatorname{gbest}^{d}-y_{j}^{d}(u)\right) \\
& +D_{2} \operatorname{rand}_{j}(u)\left(\operatorname{pbest}_{j}^{d}(u)-y_{j}^{d}(u)\right)
\end{aligned}
$$

(4)

Where $T_{j}^{d}(u)$ for the converging molecules minimizes the exponentially over time and is calculated in Eq. (4).

$$
T_{j}^{d}(u)=0.95 \times T_{j}^{d}(u-1) . \quad \cdot(5)
$$

The best previous location of the $j$ of the particle is represented as pbest $_{j}=\left(\right.$ pbest $_{j}^{1}$, pbest $_{j}^{2}, \ldots .$. pbest $\left._{j}^{m}\right)$ and the best previous location of all particles is represented as gbest $_{j}=\left(\right.$ gbest $_{j}^{1}$, gbest $_{j}^{2}, \ldots \ldots \ldots \ldots . . .$. gbest $\left._{j}^{m}\right)$. Random vectors within the corresponding ranges initialize the location and velocity of the each particle. The velocity limits used in the particles which is $\left[-v_{\min }, v_{\max }\right]$. The inertia weight $w$ reflects the resistance of the particles to the slow its movement when the $\left|v_{j}\right|>v_{\max }$ then $\left|v_{j}\right|=v_{\max }$. The randomized characteristic is provided to the search algorithm, while the uniform random variable such as $\operatorname{rand}_{j}(u)$ in the interval $[0,1]$ at the time $u$. The two acceleration constants are represented as $D_{1}$ and $D_{2}$.

The mass $m$ of each particle is a random number and it has a range $0<m \leq 1$. once the mass recognized, it remains constant throughout the execution of the whole algorithm, because it considers only one type of gas at any time. Various executions of the algorithm generated by using a random number for simulating the different type of particles.

Based on the motion equation, the location of the particle is expressed as Eq. (6),

$$
y_{u+1}^{j}=\frac{1}{2} a_{j}^{d}(u+1) u^{2}+v_{j}^{d}(u+1) u+y_{j}^{d}(u)
$$

Where acceleration of the agent is in the dimension represented as . Based on the acceleration can obtain in Eq. (7).

$$
a_{d}^{j}=\frac{\left(d v_{j}^{d}\right)}{d u}
$$

Based on the gas particles law is represented in Eq. (7).

$$
d k_{d}^{j}=\frac{1}{2} m\left(d v_{j}^{d}\right)^{2} \Rightarrow d v_{j}^{d}=\sqrt{\frac{2\left(d k_{j}^{d}\right)}{m}} \text {. }
$$

From Eq. (7) and (8), the acceleration is represented as Eq. (9).

$$
a_{d}^{j}=\frac{\sqrt{\frac{2\left(d k_{j}^{d}\right)}{m}}}{d u} \ldots
$$


The acceleration equation is re-written based on the time interval which is shown in Eq. (10).

$$
a_{d}^{j}=\frac{\sqrt{\frac{2\left(\Delta k_{j}^{d}\right)}{m}}}{\Delta u} \cdot \text {. . }
$$

In a unit time interval, the acceleration would be Eq. (11).

$$
a_{d}^{j}=\sqrt{\frac{2\left(d k_{j}^{d}\right)}{m}} \cdot \text {. . (11) }
$$

From Eq. (6) and Eq. (11), the location of the particle is computed by expressed by Eq. (12).

$$
\begin{aligned}
& y_{u+1}^{j}=\frac{1}{2} a_{j}^{d}(u+1) \Delta u^{2}+v_{j}^{d}(u+1) \Delta u+y_{j}^{d}(u) \Rightarrow \\
& y_{u+1}^{j}=\frac{1}{2} \sqrt{\frac{2\left(\Delta k_{j}^{d}\right)}{m}}(u+1) \Delta u^{2}+v_{j}^{d}(u+1) \Delta u+y_{j}^{d}(u) .
\end{aligned}
$$

At last, assuming that the molecular mass is a random number in all execution of the algorithm but it is identical for all particles in an execution. To make process easier, the location is updated periodically, which is expressed in Eq. (13).

$$
y_{u+1}^{j}=\sqrt{\frac{2\left(\Delta k_{j}^{d}\right)}{m}}(u+1)+v_{j}^{d}(u+1)+y_{j}^{d}(u) \ldots .
$$

The minimum fitness function is determined by using the following Eq. (14).

$$
\begin{array}{ll}
\text { pbest }_{j}=f\left(y_{j}\right), & \text { if } \quad f\left(y_{j}\right)<f\left(\text { pbest }_{j}\right) \\
\text { gbest } & =f\left(y_{j}\right), \quad \text { if } \quad f\left(y_{j}\right)<f(\text { gbest }) . . .
\end{array}
$$

The position of each particle is modified by using the space among the current position and the space among the current position and .

\section{A. Fuzzy C Means Algorithm}

FCM system is partitioning the dataset into number of clusters based on the following objective function Eq. (15).

$$
J_{m}=\sum_{i=1}^{c} \sum_{k=1}^{N} u_{i k}^{p}\left\|x_{k}-v_{i}\right\|^{2} \text {. . . . }
$$

Where $\mathrm{p}$ indicates the real number, which denotes the quantity controlling of the fuzziness of the resultant group, is the membership of the data point belongs to the cluster and is the pixel of the image which satisfying and is the centroid of the cluster. From the above equation 1, where is the total number of clusters and denotes the number of data points. The FCM makes the partitioning by iteratively updating the membership values and the cluster centroids. The membership value of each data point to the every centroid also derived after each time updating of centroids that can be done by the Eq. (16).

$$
u_{i k}=\frac{1}{\sum_{j=1}^{c}\left(\frac{\left\|x_{k}-v_{i}\right\|^{2}}{\left\|x_{k}-v_{j}\right\|^{2}}\right)^{\frac{1}{p-1}}}
$$

The cluster centroids are updated based on the distance between the data point to the cluster centroid which is done by the following Eq. (17).

$$
v_{k}=\frac{\sum_{k=1}^{N} x_{k} U_{i k}^{p}}{\sum_{k=1}^{N} u_{i k}^{p}} v_{i}=\frac{\sum_{j=1}^{N} u_{i j}^{m} x_{j}}{\sum_{j=1}^{N} u_{i j}^{m}} \text {. . . (17) }
$$

The objective function performs the calculation to measure the weighted sum of results between the cluster center and data presents in the fuzzy clusters. FCM provides better segmentation results for the images, which does not have any noise. However, the FCM fails to classify the noisy data as a result of the anomalies of the element data, which activates relegating the participation esteems to emerge as incorrect this is the crucial rationalization in the back of inappropriate division takes vicinity throughout the managing of a loud image with the resource of the FCM.

\section{B. Kernelized Fuzzy C means (KFCM)}

With the aim to triumph over the issue of the standard FCM calculation, the KFCM calculation is based. With the assistance of a nonlinear mapping potential, the KFCM modifications over the statistics in the image aircraft into lowering aspect dimensional difficulty region. The tough and nonlinear distinguishable problem inside the facts aircraft may be modified over with the help of the mapping ability into straight away divisible in a while place. At that factor, the FCM can play out its activity with the inferred highlight region. The aim capability of the KFCM is displayed inside the accompanying (18) condition.

$$
\begin{aligned}
& J_{m}=\sum_{i=1}^{c} \sum_{k=1}^{N} u_{i k}^{p}\left\|\varphi\left(x_{k}\right)-\varphi\left(v_{i}\right)\right\|^{2} \\
& =2 \sum_{i=1}^{c} \sum_{k=1}^{N} u_{i k}^{p}\left(1-k\left(x_{k}-v_{i}\right)\right)
\end{aligned}
$$

Here is the mapping function. Here we use the Gaussian Kernel Function (GKF) for non-linear mapping of the image plane into the linear high dimensional feature space. The GKF is presented in the following below equation (19).

$$
K(x, y)=\exp \left(-d(x, y)^{2} / \sigma^{2}\right)
$$

Substituting equation 5 into the equation 4 then the Gaussian kernel objective function is presented in the following below equation (20)

$$
\begin{aligned}
& J_{m}=\sum_{i=1}^{c} \sum_{k=1}^{N} u_{i k}^{p}\left\|\varphi\left(x_{k}\right)-\varphi\left(v_{i}\right)\right\|^{2} \\
& =2 \sum_{i=1}^{c} \sum_{k=1}^{N} u_{i k}^{p}\left(1-K\left(x_{k}, v_{i}\right)\right)
\end{aligned}
$$

Where the membership function and the updating the centroid is calculated by the following Eq 21 and 22 respectively. 


$$
\begin{aligned}
& u_{i k}=\frac{\left(1-K\left(x_{k}, v_{i}\right)\right)^{-1 /(p-1)}}{\sum_{i=1}^{c}\left(1-K\left(x_{j}, c_{i}\right)\right)^{-1 /(p-1)}} \\
& v_{i}=\frac{\sum_{k=1}^{n} u_{i k}^{p} K\left(x_{k}-v_{i}\right) x_{k}}{\sum_{k=1}^{n} u_{i k}^{p} K\left(x_{k}, v_{i}\right)}
\end{aligned}
$$

\section{B. Extreme Learning Machine}

The mapping function of KFCM method makes the input data into a high dimensional feature vector, which helps the FCM to handle the noisy image. However, high speed making ready of dimensional detail vectors requests severa emphasess, which gadgets apart greater execution attempt to make the enrollment paintings appropriate for photo department. due to the standard participation work, the comparative devices in a photo isn't assembled in a solitary parcel. to attend to this problem, we use the superb mastering calculation, which makes the enrollment capacity relying on examples within the image by using using gaining knowledge of method.

The tremendous reading machines are utilized within the ANN for purchasing ready the system. on this paper, we alter the gaining knowledge of method to expect the future estimation of the fluffy enrollment art work, which activates faded running time of the bunching method. The accompanying Eq. (23) is carried out to foresee the destiny estimation of the fluffy enrollment esteems.

$$
u_{i k}^{\wedge}=\eta\left(\frac{1}{\left(u_{i k} X\right)}\right) Y
$$

Where represents the future membership matrix and represents the existence membership matrix. The learning rate is represented by and the indicates the input matrix and specifies current partitioned matrix. The future centroid can be calculated using the following Eq (24).

$$
v_{i}^{\wedge}=\frac{\sum_{k=1}^{n} u_{i k}^{\wedge} K\left(x_{k}-v_{i}\right) x_{k}}{\sum_{k=1}^{n} u_{i k}^{\wedge} K\left(x_{k}, v_{i}\right)}
$$

\section{RESULT AND DISCUSSION}

The KGMO-KFCM-BIM framework become tested with the assistance of MATLAB reason programming version 2018 b. The entire work is finished with the resource of utilising I3 framework with 2 GB RAM. The exhibition basis is carried out via way of utilizing some T2-weighted MRI human head filters. The T2-weighted MRI head outputs had been chosen from the T1-WCEMRI database. maximum severe, cycles is implemented in KGMO grouping. This cycle is good enough to yield the great centroids for this proposed strategy. each subjective and quantitative approvals had been utilized for the presentation evaluation. The exhibition of the proposed technique changed into analyzed as a long way as bones coefficient, Jaccard co-proficient, TPR, FPR and exactness.

\section{A. usual overall performance measures}

The relationship some of the information and yield factors of a framework is understand by way of making use of the affordable execution measurements like shakers coefficient, Jaccard co-effective, TPR and FPR. In division approval, the bones coefficient is communicated concerning TP, TN, FP and FN tallies, this is received by way of the use of coordinating the portioned final consequences with floor reality photograph. the overall recipe used to determine the bones coefficient is spoken to within the Eq. (25).

$$
\text { Dicecoefficient }=\frac{2 T P}{(2 T P+F P+F N)}
$$

Where, the dice coefficient value " 0 " shows no similarity between the results and the value " 1 " demonstrates the similarity between the output and ground truth image.

In Jaccard coefficient, the TP values are detected by the overlaps between the manually segmented ground truth tumor labels and the machine generated tumor labels. The general formula used to calculate Jaccard co-efficient is denoted in the Eq. (26).

$$
\text { Iaccardcoefficient }=\frac{T P}{F P+F N+T P}
$$

What's more, exactness is the reasonable assessment measurements for finding the adequacy of ordinariness and variation from the norm of mind tumor location. These measures are factual changeability and a depiction of irregular blunders. The general recipe of precision for deciding the typicality and variation from the norm of mind tumor location is spoken to in the Eq. (27)

$$
\text { Accuracy }=\frac{T P+T N}{T P+T N+F P+F N} \times 100
$$

wherein, is spoken to as obtrusive remarkable, is indicated as fake tremendous, is expressed as obvious poor and is determined as faux horrific.

\section{A. Experimental end result on T1-WCEMRI dataset}

In this test studies, T1-WCEMRI dataset is surveyed for contrasting the shakers and Jaccard coefficient execution of FCM and GA-KFCM and the KGMO-KFCM-BIM it's appeared in the desk 1. The T1-WCEMRI dataset incorporates 3064 pix with three education of thoughts pictures: meningioma, glioma and pituitary tumor. The exhibition assessment of some instance snap shots are regarded within the Fig. 2. 

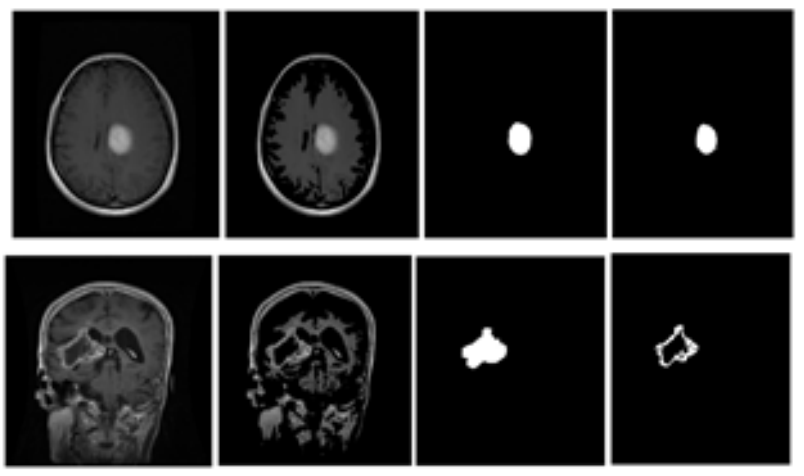

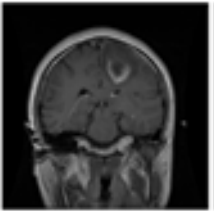

(a)

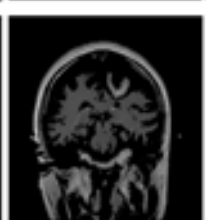

(b)

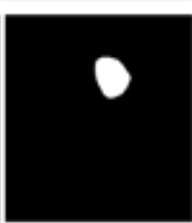

(c)

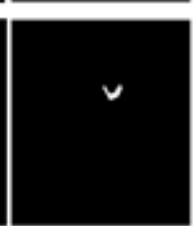

(d)
Fig. 2. (a) Input image, (b) cluster image, (c) Ground image, (d) Output image

TABLE I. DICE AND JACCARD COEFFICIENT COMPARISON OF FCM AND GA-KFCM AND THE KGMO-KFCM-BIM

\begin{tabular}{|l|l|l|l|l|}
\hline Class & Clustering technique & $\begin{array}{c}\text { Dice } \\
\text { coefficien } \\
\boldsymbol{t}\end{array}$ & $\begin{array}{c}\text { Jaccard } \\
\text { coefficien } \\
\boldsymbol{t}\end{array}$ & $\begin{array}{c}\text { Accurac } \\
\boldsymbol{y}\end{array}$ \\
\hline \multirow{3}{*}{$\begin{array}{l}\text { Meningiom } \\
\text { Glioma }\end{array}$} & FCM & 0.26158 & 0.1625 & 94.1253 \\
\cline { 2 - 5 } & GA-KFCM & 0.39485 & 0.2460 & 95.6818 \\
\cline { 2 - 5 } & $\begin{array}{l}\text { KGMO-KFCM-BI } \\
\text { M }\end{array}$ & 0.4075 & 0.3041 & 96.4890 \\
\cline { 2 - 5 } & FCM & 0.4765 & 0.2511 & 93.7741 \\
\cline { 2 - 5 } & GA-KFCM & 0.6879 & 0.4050 & 94.7245 \\
\cline { 2 - 5 } & $\begin{array}{l}\text { KGMO-KFCM-BI } \\
\text { Pituitary }\end{array}$ & 0.92380 & 0.88347 & 96.9931 \\
& FCM & 0.5847 & 0.3570 & 97.0235 \\
\cline { 2 - 5 } & GA-KFCM & 0.6147 & 0.38217 & 98.0087 \\
\cline { 2 - 5 } & $\begin{array}{l}\text { KGMO-KFCM-BI } \\
\text { M }\end{array}$ & 0.7258 & 0.6010 & 98.8912 \\
\hline
\end{tabular}

From the desk 1, examination demonstrates that the KGMO-KFCM-BIM technique supply lots better effects. The everyday of the KGMO-KFCM-BIM method is dice coefficient is zero.6857 and everyday of Jaccard coefficient is 0.5962. FCM and GA-KFCM conveys zero.4409 and 0.5658 of bones coefficient. Likewise, ordinary Jaccard coefficient of the FCM and GA-KFCM strategies are zero. 2569 and zero.3444. The regular precision of the FCM and GA-KFCM are 90 four.9743 and 96.1383. consequently, the KGMO-KFCM-BIM technique supply 90 seven.4577 of exactness it's higher even as contrasted with extraordinary modern-day frameworks.

\section{A. Comparative research}

desk 2 and Fig. three. provides the close to research of modern art work and the proposed paintings execution. $\mathrm{H}$. Ali, M. Elmogy, et al. [39] proposed every different mind image department framework, which trusted morphological pyramid with FCM grouping. in the beginning, a wavelet multi-dreams method modified into created on the way to maintain up spatial setting between the pixels. At that element, morphological pyramid come to be used to build the sharpness of mind snap shots. At very last, branch grow to be conveyed utilizing FCM grouping. The trial stop result demonstrates that the created approach performed $96 \%$ of order precision. what is greater, E. Abdel-Maksoud, M.
Elmogy, et al. [40] proposed a move breed bunching technique for programmed mind tumor department. The proposed method became the combination of good enough-implies grouping and FCM for image department inside the components of insignificant calculation time. The examination emerge as finished on freely available databases: brain (DS1), (DS2), and (DS3) in an effort to approve the proposed approach precision. The created process finished 96.eighty three\% of affiliation exactness. even though, the proposed work accomplishes ninety seven. $4577 \%$ of precision that became higher than the cutting-edge works.

TABLE II. COMPARATIVE ANALYSIS OF PROPOSED AND EXISTING METHODOLOGIES

\begin{tabular}{|l|l|l|l|l|}
\hline Class & Clustering technique & $\begin{array}{c}\text { Dice } \\
\text { coefficien } \\
\boldsymbol{t}\end{array}$ & $\begin{array}{c}\text { Jaccard } \\
\text { coefficien } \\
\boldsymbol{t}\end{array}$ & $\begin{array}{c}\text { Accurac } \\
\boldsymbol{y}\end{array}$ \\
\hline \multirow{3}{*}{$\begin{array}{c}\text { Meningiom } \\
\mathbf{5}\end{array}$} & FCM & 0.26158 & 0.1625 & 94.1253 \\
\cline { 2 - 5 } & GA-KFCM & 0.39485 & 0.2460 & 95.6818 \\
\cline { 2 - 5 } & $\begin{array}{l}\text { KGMO-KFCM-BI } \\
\text { Glioma }\end{array}$ & 0.4075 & 0.3041 & 96.4890 \\
& FCM & 0.4765 & 0.2511 & 93.7741 \\
\cline { 2 - 5 } & GA-KFCM & 0.6879 & 0.4050 & 94.7245 \\
\cline { 2 - 5 } & $\begin{array}{l}\text { KGMO-KFCM-BI } \\
\text { P }\end{array}$ & 0.92380 & 0.88347 & 96.9931 \\
\hline \multirow{5}{*}{ Pituitary } & FCM & 0.5847 & 0.3570 & 97.0235 \\
\cline { 2 - 5 } & GA-KFCM & 0.6147 & 0.38217 & 98.0087 \\
\cline { 2 - 5 } & $\begin{array}{l}\text { KGMO-KFCM-BI } \\
\text { M }\end{array}$ & 0.7258 & 0.6010 & 98.8912 \\
\hline
\end{tabular}

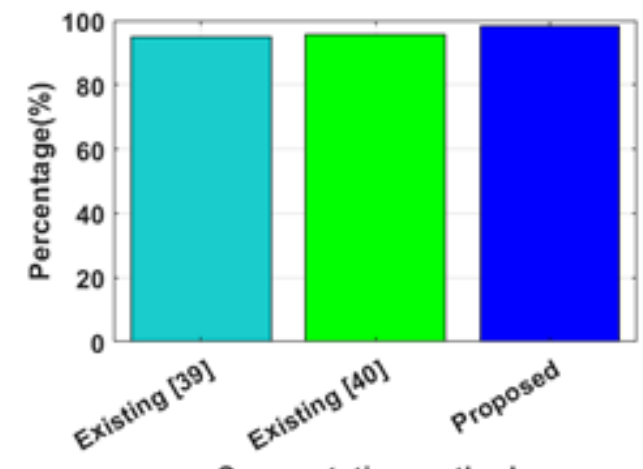

Segmentation methods

Fig. 3. Average accuracy comparison of KGMO-KFCM-BIM and existing methodologies

\section{CONCLUSION}

Cerebrum tumor identity is one of the most noteworthy research assignment in pc helped fitness staring at framework. The intention of the test is to build up a valid department method for fragmenting the sound and tumor area of thoughts making use of T1-WCEMRI database. The KGMO-KFCM-BIM art work is a singular centroid instatement method depending on KGMO for introducing the FCM bunching to element the MRI of head filters. The results demonstrates that the proposed advent along 21 cycles are ok to yield the proper effects. The test results demonstrates that the KGMO-KFCM-BIM method gives higher consequences and it is steadily sturdy against indicates FCM. this is beneficial to boost up the mind demonstrative framework

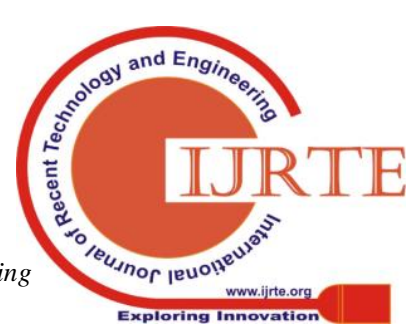


relying upon cerebrum tissue department method.

\section{REFERENCES}

1. S. Pilleron, D. Sarfati, M.Janssen-Heijnen, J. Vignat, J. Ferlay, F. Bawl, and i.Soerjomataram, "international malignant growth prevalence in more set up grown-ups, 2012 and 2035," A populace-based completely look at. worldwide diary of disorder, July 2018.

2. J. Ferlay, I. Soerjomataram, R. Dikshit, S. Eser, C.Mathers, M. Rebelo, D. M. Parkin, D. Forman, and F. Bawl, "Malignancy incidence and mortality around the arena: property, techniques and massive examples in GLOBOCAN 2012," international diary of sickness, vol. 136, no. 5, pp. E359-E386. 2015

3. R. Thambi, and S. Kandamuthan, "Histopathological evaluation of brain Tumors-A Seven 12 months examine from a Tertiary Care middle in South India," magazine of clinical and indicative research: JCDR, vol. 11, no. 6, pp EC05. June 2017.

4. M. Lacroix, D. Abi-said, D. R. Fourney ZL. Gokaslan, W. Shi, F. DeMonte, FF Lang, IE. McCutcheon, SJ. Hassenbusch, E. Holland, and k. Hess, "A multivariate exam of 416 patients with glioblastoma multiforme: forecast, degree of resection, and survival," magazine of neurosurgery, vol.90 5, no. 2, pp.a hundred ninety-198. August 2001.

5. N. Sanai, MY. Polley, MW. McDermott, AT. Parsa, and MS. Berger, "A diploma of resection restriction for these days analyzed glioblastomas," journal of neurosurgery, vol. 115, no. 1, pp.three-8. July 2011.

6. MJ. McGirt, D. Mukherjee, KL. Chaichana, KD. Than, JD. Weingart, and A.Quinones-Hinojosa, "courting of exactly procured engine and language deficiencies on with the aid of and massive survival after resection of glioblastoma multiforme," Neurosurgery, vol. sixty 5, no. three, pp. 463-470, SEPTEMBER 2009.

7. C. Kut, KL. Chaichana, J. Xi, SM. Raza, X. Ye, ER. McVeigh, FJ. Rodriguez, A. Quiñones-Hinojosa, and X. $\mathrm{Li}$, "Discovery of human cerebrum malignancy invasion ex vivo and in vivo making use of quantitative optical intelligence tomography," technology translational prescription, vol.7, no. 292, pp. 292ra100-292ra100, June 2015.

8. OM. Rygh, T. Selbekk, SH. Torp, S. Lydersen, TAN. Hernes, and G. Unsgaard, "examination of explored three-d ultrasound discoveries with histopathology in consequent durations of glioblastoma resection," Acta neurochirurgica, vol. 150, no. 10, p.1033, September 2008.

9. M. Ji, DA. Orringer, CW. Freudiger, S. Ramkissoon, X. Liu, D. Lau, AJ. Golby, I. Norton, M. Hayashi, the huge apple. Agar, and GS. more youthful, "speedy, mark loose identity of cerebrum tumors with energetic Raman dissipating microscopy," science translational remedy, vol. 5, no. 201, pp. 201ra119-201ra119, September 2013.

10. adequate. Özduman, E. Yıldız, A. Dinçer, A. Sav, and MN. Pamir, "making use of intraoperative particular complexity upgraded T1-weighted MRI to apprehend last tumor in glioblastoma scientific system," mag of neurosurgery, vol. a hundred and twenty, no. 1, pp.60-sixty six, January 2014.

11. S .Zausinger, B.cheder, E. Uhl, T. Heigl, D. Morhard, and JC. Tonn, "Intraoperative figured tomography with incorporated path framework in spinal adjustments," backbone, vol. 34, no. 26, pp.2919-2926, 2009.

12. X. Muñounces, J. Freixenet, X. Cufi, and J. Mart1, "Methodologies for picture department consolidating district and restriction data," sample acknowledgment letters, vol. 24, no. 1, pp.375-392, 2003.

13. S. Krinidis, and V. Chatzis, "A complete of lifestyles fluffy nearby data C-implies grouping calculation," IEEE Transactions on picture Processing, vol.19, no. 5, pp. 1328-1337, can also 2010.

14. J. Shi, and J. Malik, "Standardized cuts and photo department," IEEE Transactions on example research and system insight, vol. 22, no. eight, pp.888-905, August 2000.

15. M. Andersson, J. Gudmundsson, and C. Levcopoulos, "Surmised separation prophets for charts with thick organizations," Lecture notes in software engineering, vol. 3341, pp.fifty three-sixty four. August 2004.

16. G. Govaert, and M. Nadif, "An EM calculation for the rectangular blend version," IEEE Transactions on pattern evaluation and device know-how, vol. 27, no. 4, pp.643-647, April 2005.

17. XF. Wang, and DS. Huang, "A story thickness primarily based grouping form by way of utilising stage set strategy," IEEE Transactions on mastering and facts constructing, vol. 21, no. eleven, pp.1515-1531, information bunching utilising bacterial rummaging development 2009

18. J. Zhang, and J. Kerekes, "a flexible thickness based totally completely version for isolating ground comes decrease again from photon-checking laser altimeter statistics," IEEE Geoscience and far off Sensing Letters, vol. 12, no. 4, pp.726-730, April 2015.

19. M. Ester, HP Kriegel, J. Sander, and X. Xu, "A thickness based totally totally calculation for finding bunches in huge spatial databases with commotion," In Kdd, vol. ninety six, no. 34, pp. 226-231, August 1996.

20. A. Omrani, and okay. Santhisree, "Bunching consecutive information with OPTICS," In, In lawsuits IEEE 0.33 international convention on communique software program and Networks (ICCSN), pp. 591-594, might also 2011.

21. M. De Marsico, M.Nappi, D. Riccio, and H. Wechsler, "lively face acknowledgment for uncontrolled posture and brightening modifications," IEEE Transactions On systems, guy, And Cybernetics: structures, vol. forty 3 , no.1, pp.149-163, 2013.

22. W. Sheng, S. Chen, G. Xiao, J. Mao, and Y. Zheng, "A biometric key age approach relying on semisupervised information grouping," IEEE Transactions on structures, man, and Cybernetics: systems, vol. 45,no. nine, pp.1205-1217, 2015.

23. L. Zhu, FL. Chung, and S.Wang, "Summed up fluffy c-implies grouping calculation with improved fluffy parcels," IEEE Transactions on structures, guy, and Cybernetics, issue B (Cybernetics), vol. 39, no. three, pp.578-591, June 2009.

24. JC. Bezdek, "instance reputation with Fuzzy objective function Algorithms," Kluwer instructional Publishers, Norwell, MA, u.s., 1981

25. Y. Yang, C. Zheng, and P. Lin, "Fluffy C-implies grouping calculation with a unique punishment term for photograph branch," Optoelectronics assessment, vol thirteen, no. 4, p.309. 2005.

26. M. Girolami, "Mercer element based bunching in spotlight area," IEEE Transactions on Neural Networks, vol. thirteen, no. 3, pp.780-784, 2002.

27. S. Chen, and D. Zhang, "Hearty picture department making use of FCM with spatial imperatives relying on new aspect initiated separation measure," IEEE Transactions on systems, guy, and Cybernetics, part B (Cybernetics), vol. 34, no. four, pp.1907-1916, August 2004. 
28. U. Maulik, and S. Bandyopadhyay, "Hereditary calculation primarily based grouping machine," pattern acknowledgment, vol. 33, no. nine, pp. 455-1465, April 2000.

29. okay. Premalatha, and AM. Natarajan, "a few other technique for information bunching relying on PSO with close by seek," pc and information technology, vol. 1, no. four, p.139, November 2008.

30. M. Wan, L. Li, J. Xiao, C.Wang, and Y Yang, "statistics grouping utilizing bacterial scrounging improvement," magazine of intelligent statistics structures, vol. 38,no. 2, pp.321-341 April 2012.

31. SZ. Selim, and k. Alsultan, "A mimicked tempering calculation for the grouping issue," pattern acknowledgment, vol. 24, no. 10, pp. 1003-1008, January 1991.

32. C. Zhang, D. Ouyang, and J. Ning, "A faux honey bee province technique for grouping," professional systems with programs, vol. 37, no. 7, pp. 4761-4767, July 2010.

33. J. Senthilnath, SN. Omkar, and V. Mani, "Grouping using firefly calculation: execution observe," Swarm and Evolutionary Computation, vol. 1, no. three, pp. 164-171, September 2011.

34. R. Shang, P. Tian, L. Jiao, R. Stolkin, J. Feng, B. Hou, and X. Zhang, "A spatial fluffy bunching calculation with piece metric depending on insusceptible clone for SAR photograph division," IEEE journal of determined on topics in implemented Earth Observations and a ways flung Sensing, vol. 9, no. four, pp. 1640-1652, April 2016.

35. L. Liu, A. Yang, W. Zhou, X. Zhang, M. Fei, and X. Tu, "robust dataset association method relying on neighbor searching and piece fluffy c-implies," IEEE/CAA magazine of Automatica Sinica, vol. 2, no. three, pp. 235-247, July 2015.

36. A. Elazab, C. Wang, F. Jia, J. Wu, G. Li, and Q. Hu, "department of cerebrum tissues from appealing reverberation pics using adaptively regularized piece primarily based fluffy strategies grouping," Computational and clinical strategies in medicine, 2015.

37. YT. Chen, "Medicinal picture Segmentation the usage of impartial issue evaluation-based Kernelized Fuzzy-way Clustering," Mathematical troubles in Engineering, January 2017.

38. H. Li, H. He, and Y. Wen, "Dynamic molecule swarm streamlining and okay-implies grouping calculation for picture department," Optik - global journal for mild and Electron Optics, vol. 126, no. 24, pp. 4817-4822, December 2015.

39. H. Ali, M. Elmogy, E. El-Daydamony, and A. Atwan, "Multi-dreams MRI cerebrum image department depending on morphological pyramid and fluffy c-mean bunching," Arabian magazine for era and Engineering, vol.40, no.eleven, pp. 3173-3185, November 2015.

40. E. Abdel-Maksoud, M. Elmogy, and R. Al-Awadi, "Cerebrum tumor department depending on a half breed bunching technique," Egyptian Informatics magazine, vol.16, no.1, pp. seventy one-eighty one, March 2015. 